\title{
EQUIVALENCE OF ACCELERATED 10-DAYS AND STANDARD OF DEEP TRANSCRANIAL MAGNETIC STIMULATION WITH H1- COIL PROTOCOLS IN TREATMENT RESISTANT DEPRESSION; A PILOT STUDY
}

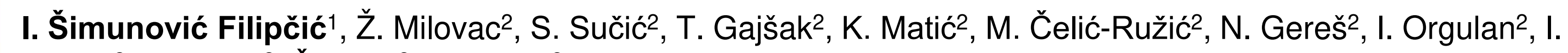

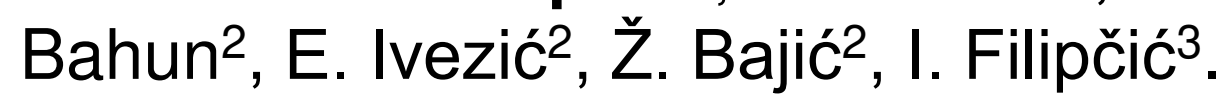

${ }^{1}$ Department of Psychiatry and Psychological Medicine, University Hospital Center Zagreb;2Psychiatric Hospital "Sveti Ivan" 3 Faculty of Dental Medicine and Health- Josip Juraj Strossmayer University of Osijek and School of Medicine, University of Zagreb, Croatia

Objective: To test the efficacy and safety equivalence of accelerated 10-days and standard daily deep transcranial magnetic stimulation protocols (dTMS) with $\mathrm{H} 1$-coil in treatment-resistant depression (TRD). Background: dTMS is an effective option for TRD but requires 20-days repeated treatments. Accelerated dTMS, delivered over the shorter period, would provide important advantages.

Materials and methods: We performed the pilot equivalence trial with blinded and independent outcome assessment. A total of 16 TRD patients received a twice-daily 20 minutes dTMS treatment over 10 consecutive weekdays (the total of 36000 pulses). Historic control group was obtained from our previous RCT $(n=49)^{1}$. The primary outcome was change in the Hamilton depression scale (HAM-D17). We set the equivalence margin to $+/-3$ points of the change in total HAM-D17 score.

Table 1. Participants baseline characteristics in intention-to-treat

\begin{tabular}{lcc}
\hline population & \multicolumn{2}{c}{ Protocol } \\
\cline { 2 - 3 } & $\begin{array}{c}\text { Accelerated } \\
(\mathrm{n}=27)\end{array}$ & $\begin{array}{c}\text { Standard } \\
(\mathrm{n}=29)\end{array}$ \\
\hline Gender & $13(48)$ & $14(48)$ \\
$\quad$ man & $14(52)$ & $15(52)$ \\
$\quad$ women & $55(52-60)$ & $46(41-56)$ \\
Age (years), median (IQR) & & \\
Education & $21(78)$ & $19(66)$ \\
$\quad$ primary or secondary & $6(22)$ & $10(34)$ \\
$\quad$ university & & \\
Work status & $18(67)$ & $21(72)$ \\
$\quad$ unemployed or retired & $9(33)$ & $8(28)$ \\
$\quad$ employed & $28(24-31)$ & $28(22-30)$ \\
Body mass index (kg/m $\left.{ }^{2}\right)$, median (IQR) & $14(52)$ & $16(57)$ \\
Current smokers & $3(2-4)$ & $3(2-4)$ \\
Number of household members, median (IQR) & & \\
Clinical characteristics & & \\
Diagnosis & $4(15)$ & $7(24)$ \\
$\quad$ depressive episode (F32) & $23(85)$ & $22(76)$ \\
$\quad$ recurrent depression (F33) & $10(4-16)$ & $10(5-17)$ \\
Duration of MDD (years), median (IQR) & & \\
Clinical global impression - severity scale & $17(65)$ & $23(82)$ \\
$\quad$ not severely ill (?5) & $9(35)$ & $5(18)$ \\
$\quad$ severely ill (6,7) & & \\
Treatment with antidepressants & $9(22)$ & $11(38)$ \\
$\quad$ SSRI & $6(37)$ & $12(41)$ \\
$\quad$ SNRI & $0(0)$ \\
$\quad$ NaSSA & $6(21)$ \\
$\quad$ other antidepressants & &
\end{tabular}

Results: All patients completed the study, and no patient had a serious adverse event. Unadjusted mean reduction (C195\%) in HAM-D17 was identical in both protocols: -14 (-16 to $-12 ;-68 \%)$. After the adjustment for baseline HAM-D17, MDD duration, age and gender, the mean (CI95\%) change in HAMD17 was -13 (-16 to $-11 ;-64 \%)$ in accelerated, and $14(-16$ to $-13 ;-68 \%)$ in the standard protocol. The accelerated protocol $\mathrm{C} 195 \%$ was within the predefined margin $(-17$ to -11$)$ so the equivalence was established.

Crude, unadjusted analysis

Adjusted for HAM-D17 at baseline Fully adjusted

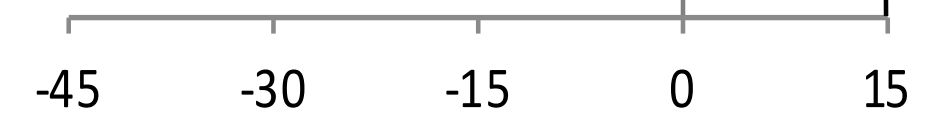

Relative change in accelerated minus relative change in standard

Figure 1. Non-inferiority analysis of accelerated standard dTMS protocol; non-inferiority margin $>15 \%$; Fully adjusted for baseline HAMD-17 score, age, gender, education, and treatment with SSRI

Conclusions: Accelerated dTMS with $\mathrm{H} 1$-coil regimen twice daily during ten days was equally safe and effective as the standard 20-days protocol for the treatment for TRD. More research is recommended on the drug-naïve patients to control the confounding effect of duration of treatment with antidepressants which may promote the hypothesis of inferiority of accelerated protocols. 